\title{
Symmetric Instantons and the ADHM Construction
}

\author{
Gil Bor ${ }^{1}$, Jan Segert ${ }^{2 \star \star}$
}

1 CIMAT, A.P. 402, Guanajuato 36000, Guanajuato, Mexico. E-mail address: gil@fractal.cimat.mx

2 Department of Mathematics, University of Missouri, Columbia, MO 65211, USA. E-mail address: jan@math.missouri.edu

Received: 13 June 1994/Accepted: 27 June 1996

\begin{abstract}
We construct all $S U(2)$ Yang-Mills instantons on $S^{4}$ that admit a certain symmetry ("quadrupole symmetry"). This is accomplished by an equivariant version of the "ADHM monad" classification of instantons. This work is part of an attempt to better understand the structure of non-self-dual Yang-Mills connections with the same symmetry.
\end{abstract}

\section{Introduction}

A. Statement of Results. An instanton, in this paper, refers to a unitary connection with anti-self-dual curvature on a rank-two hermitian vector bundle over the standard four-sphere $S^{4}$. Such a bundle is determined, up to an isomorphism, by its second Chern number $c_{2}$, and admits instantons if and only if $c_{2} \geqq 0$. For a detailed account of the theory of instantons on $S^{4}$, see for example the book [5].

This article is devoted to the study of instantons with "quadrupole symmetry" [4]. To define these, let the orthogonal group $S O(3)$ act on $S^{4} \subset \mathbf{R}^{5}$ via its irreducible linear representation on $\mathbf{R}^{5}$ (conjugation of traceless symmetric $3 \times 3$ real "quadrupole" matrices). Then a bundle with quadrupole symmetry consists of a rank-two hermitian vector bundle over $S^{4}$ together with a lift of the $S O(3)$-action on $S^{4}$ to a unitary action on the bundle. In general, to construct such lifts, one needs to pass from $S O(3)$ to its double-cover $\operatorname{Spin}(3) \cong S U(2)$. Finally, an instanton with quadrupole symmetry, or simply a symmetric instanton, consists of a bundle with quadrupole symmetry together with an instanton connection which is invariant under the $S U(2)$-action on the bundle.

The classification of bundles with quadrupole symmetry is quite simple, given by a pair of odd positive integers $\left(n_{+}, n_{-}\right)$. The significance of these integers is the following: the singular locus of the $S U(2)$-action on $S^{4}$ consists of exactly two orbits; for a point on one of these orbits the identity component of the stabilizer subgroup is a circle group which acts on the fiber with weights $\left\{n_{+},-n_{+}\right\}$or



Part of this work was done at the 1991 Regional Geometry Institute in Park City, Utah 
$\left\{n_{-},-n_{-}\right\}$, depending on the orbit. In terms of these invariants, the second Chern number of the corresponding bundle is

$$
c_{2}=\left(n_{+}^{2}-n_{-}^{2}\right) / 8
$$

More details on "quadrupole symmetry" can be found in the Appendix at the end of this article or in the articles [20] and [10]. In these two articles it was also shown that there are no symmetric instantons on bundles with $n_{-} \geqq 3$.

Here, we address the question for the remaining cases of symmetry type $\left(n_{+}, 1\right)$. We obtain a constructive classification of all instantons with quadrupole symmetry. The main result is the following:

Theorem 1.1. There exists exactly one symmetric instanton on each of the bundles over $S^{4}$ with quadrupole symmetry of type $\left(n_{+}, 1\right)$, where $n_{+}$is an odd positive integer. Furthermore, the corresponding symmetric ADHM monads can be explicitly constructed (see below).

To explain the method of our proof, let us recall that the classification of instantons was accomplished in two steps. First, a "twistor transform" provided a bijective correspondence between instantons and a class of holomorphic bundles over $\mathbb{C} P^{3}$ [24], and second, the Atiyah-Drinfeld-Hitchin-Manin construction [2] provided a bijective correspondence between this class of holomorphic bundles and "ADHM monads" (see Sect. 3 for a review). We summarize these correspondences in the following diagram:



Now, the functorial nature of these correspondences implies that the ADHM monads corresponding to instantons with quadrupole symmetry naturally admit an $S U(2)$-action, so the proof of Theorem 1.1 amounts to the construction of all "ADHM monads with quadrupole symmetry." To construct these, we use an equivariant index theorem to determine the $S U(2)$-action on these ADHM monads (Sect.4), and then construct all these ADHM monads with quadrupole symmetry (Sects. 5 and 6).

Some technical aspects of our proof, such as Proposition 5.3, may be of independent interest for the theory of $S U(2)$-representations and Clebsch-Gordan coefficients.

The results in this paper can be obtained by different methods. Dimensional reduction and ordinary differential equations techniques yield an alternate proof [18] of the existence statement of Theorem 1.1, but do not provide an explicit construction of the symmetric instantons. For any $n_{+}$, an equivariant version of the twistor transform provides a bijective correspondence between symmetric instantons and solutions of a certain system of algebraic equations [19]. For $n_{+}$small, the algebraic equations can be readily solved, yielding an explicit construction of the 
corresponding symmetric instanton. Other authors have previously considered equivariant ADHM constructions for certain Abelian symmetry groups [6, 7, 8, 12, 13].

B. Background. Our interest in instantons with quadrupole symmetry stems from a comparison of the theory of instantons with that of general solutions of the YangMills equations. The latter are the variational equations for the "Yang-Mills action" (the $L_{2}$-norm of the curvature), and the self-dual and anti-self-dual connections are special solutions, corresponding to the minimal critical points of the action. The general solutions to the Yang-Mills equations (not necessarily self-dual or antiself-dual) are rather poorly understood at present. For several years, a number of results $[9,23]$ and similarity with other problems (e.g., harmonic maps $S^{2} \rightarrow S^{2}$ ) suggested that every solution of the Yang-Mills equations over $S^{4}$ is either selfdual or anti-self-dual. Then in 1989, L. Sibner, R. Sibner, and K. Uhlenbeck [22] published a variational existence proof for non-self-dual solutions to the YangMills equations on the trivial bundle $\left(c_{2}=0\right)$. After that discovery we looked for other non-self-dual solutions and obtained the following result (compare with Theorem 1.1):

Theorem 1.2 ([20, 10]). There exists a symmetric connection which is a solution to the Yang-Mills equations but is neither self-dual nor anti-self-dual, on each of the bundles over $S^{4}$ with quadrupole symmetry of type $\left(n_{+}, n_{-}\right)$, where $n_{+}$and $n_{-}$ are odd positive integers $\geqq 3$.

Thus, according to this theorem and Formula (1.1), non-self-dual solutions to the Yang-Mills equations exist for all vector bundles over $S^{4}$ except possibly for those with $c_{2}= \pm 1$. (This last case remains open to date, to our knowledge). Unlike in the self-dual case, no solutions have been constructed explicitly, although numerical approximations to some of these solutions have been obtained [21].

Now, the twistor transform for instantons has a less familiar analogue for the general solutions of the Yang-Mills equations. This so-called "ambitwistor transform" provides a correspondence between Yang-Mills connections and a class of holomorphic vector bundles over the variety $F_{1,3}$ of $(1,3)$-flags in $\mathbb{C}^{4}$. We refer to [16] for details and references. One of the difficulties of this approach is the absence of an analogue of ADHM monads, so there is no known method for constructing the appropriate holomorphic bundles over $F_{1,3}$. This is summarized in the following diagram (to be compared with diagram 1.2):

YM connections on $S^{4}$
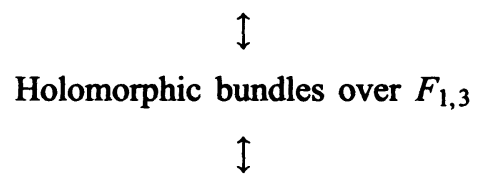

??

It is plausible that an equivariant ambitwistor transform for the non-self-dual Yang-Mills connections of Theorem 1.2 is simpler than the general ambitwistor transform without symmetry. Our motivation for studying the analogous equivariant ADHM construction in this paper is to develop an understanding of the type of simplification that may occur in the equivariant ambitwistor setting. 
Instantons with quadrupole symmetry are also interesting from a rather different and recent point of view. There is a link between such instantons and isomonodromic deformations of linear ordinary differential equations, the Painlevé transcendents, etc. This will be expounded in an upcoming article by the first author. This circle of ideas has also been recently used to study Einstein metrics with symmetry [14].

J.S. wishes to acknowledge helpful discussions or communications with R.L. Bryant, A.D. Helfer, and L.A. Sadun.

\section{2. $S U(2)-R e p r e s e n t a t i o n s$}

In this section we review some facts, mostly standard, pertaining to the representation theory of the group $S U(2)$.

Let $S U(2)$ act on $\mathbb{C}^{2}$ via the fundamental representation. Then $S U(2)$ acts in the usual way on the vector space $\mathscr{V}:=\mathbb{C}[x, y]$ of polynomials on $\mathbb{C}^{2}$. The $(d+1)-$ dimensional subspace $\mathscr{V}_{d} \subset \mathscr{V}$ of degree-d homogeneous polynomials is invariant and irreducible. Any complex irreducible finite-dimensional representation of $S U(2)$ is isomorphic to $\mathscr{V}_{d}$ for some non-negative integer $d$.

The subgroup of diagonal matrices in $S U(2)$ consists of matrices with $(z, \bar{z})$ on the diagonal, where $|z|=1$. This subgroup acts on the monomials by $x^{m} y^{n} \mapsto$ $z^{m-n} x^{m} y^{n}$, which easily yields the character $\chi_{d}$ of $\mathscr{V}_{d}$ :

$$
\chi_{d}=z^{d}+z^{d-2}+\cdots+z^{-d}=\frac{z^{d+1}-\bar{z}^{d+1}}{z-\bar{z}} .
$$

Next, we consider real and quaternionic structures. These are induced on the symmetric algebra $\mathscr{V}=S^{*}\left(\mathscr{V}_{1}\right)$ from the quaternionic representation $\mathscr{V}_{1}$, i.e. $\mathscr{V}_{1} \cong$ $\left(\mathbb{C}^{2}\right)^{*}$ admits an $S U(2)$-equivariant anti-linear endomorphism $\sigma$, satisfying $\sigma^{2}=-1$ (a "structure map"), given by

$$
\sigma: a x+b y \mapsto \bar{b} x-\bar{a} y .
$$

The induced structure map on $\mathscr{V}$ is then real $\left(\sigma^{2}=1\right)$ on the even part

$$
\mathscr{V}_{+}:=\mathscr{V}_{0} \oplus \mathscr{V}_{2} \oplus \mathscr{V}_{4} \oplus \cdots,
$$

and quaternionic $\left(\sigma^{2}=-1\right)$ on the odd part

$$
\mathscr{V}_{-}:=\mathscr{V}_{1} \oplus \mathscr{V}_{3} \oplus \mathscr{V}_{5} \oplus \cdots
$$

The structure map on monomials is given by

$$
\sigma\left(x^{m} y^{n}\right)=(-1)^{m} x^{n} y^{m}
$$

and on a general $v \in \mathscr{V}$ by anti-linear extension.

The remaining structures on $\mathscr{V}$ are conveniently described in terms of the "transvectants" (Überschiebungen) of classical invariant theory. We adopt the notation of Bryant [11]. For a non-negative integer $p$, the $p^{\text {th }}$ transvectant is the $S U(2)$-equivariant $\mathbb{C}$-bilinear map $\mathscr{V} \times \mathscr{V} \rightarrow \mathscr{V}$ defined by

$$
\langle u, v\rangle_{p}:=\frac{1}{p !} \sum_{k=0}^{p}(-1)^{k}\left(\begin{array}{l}
p \\
k
\end{array}\right) \frac{\partial^{p} u}{\partial x^{p-k} \partial y^{k}} \frac{\partial^{p} v}{\partial x^{k} \partial y^{p-k}}
$$


Remark. Here is a quick proof for the equivariance of the transvectants, as well as an "explanation" of the origin of Formula (2.3). Consider the $S U(2)$-representation $\mathbb{C}^{2} \oplus \mathbb{C}^{2}$ with linear coordinates $x, y, x^{\prime}, y^{\prime}$. Then the differential operator

$$
D:=\frac{\partial}{\partial x} \frac{\partial}{\partial y^{\prime}}-\frac{\partial}{\partial y} \frac{\partial}{\partial x^{\prime}}
$$

is $S U(2)$-invariant. This follows from the $S L(2, \mathbb{C})$-invariance of the area form $\frac{\partial}{\partial x} \wedge$ $\frac{\partial}{\partial y}$ on $\left(\mathbb{C}^{2}\right)^{*}$. Now the $p^{\text {th }}$ transvectant applied to a pair of polynomials $u$ and $v$ in $\mathscr{V}$ is given by first applying $D^{p} / p$ ! to $u \otimes v$ (thought of as the polynomial $u(x, y) v\left(x^{\prime}, y^{\prime}\right)$ on $\left.\mathbb{C}^{2} \oplus \mathbb{C}^{2}\right)$ followed by polynomial multiplication $\mathscr{V} \otimes \mathscr{V} \rightarrow \mathscr{V}$.

The transvectants induce a bilinear form and hermitian inner product on $\mathscr{V}$ as follows. The restriction of the $p^{\text {th }}$ transvectant to $\mathscr{V}_{p} \times \mathscr{V}_{p}$ is an invariant bilinear form, and by taking direct sums we obtain an invariant bilinear form on $\mathscr{V}$, denoted by $\langle\cdot, \cdot\rangle$. This form is symmetric on $\mathscr{V}_{+}$and anti-symmetric on $\mathscr{V}_{-}$; in fact, using Formula (2.3), for any $u, v \in \mathscr{V}$,

$$
\langle u, v\rangle_{p}=(-1)^{p}\langle v, u\rangle_{p} .
$$

The transvectants are compatible with the structure map $\sigma$,

$$
\sigma\langle u, v\rangle_{p}=\langle\sigma u, \sigma v\rangle_{p}
$$

for any $u, v \in \mathscr{V}$, hence the hermitian inner product on $\mathscr{V}$ defined by

$$
(u, v):=\langle\sigma u, v\rangle
$$

is $S U(2)$-invariant. The monomials $x^{m} y^{n}$ form an orthogonal basis, and

$$
\left\|x^{m} y^{n}\right\|^{2}:=\left(x^{m} y^{n}, x^{m} y^{n}\right)=m ! n !>0 .
$$

This verifies the positive-definitiveness of the hermitian inner product $(\cdot, \cdot)$, and consequently, the non-degeneracy of the bilinear form $\langle\cdot, \cdot\rangle$.

Remark. The existence of a non-degenerate invariant bilinear form implies that each of the irreducible $S U(2)$-representations $\mathscr{V}_{d}$ is isomorphic to its dual, $\mathscr{V}_{d} \cong$ $\left(\mathscr{V}_{d}\right)^{*}$.

Tensor products of the irreducible representations $\mathscr{V}_{d}$ decompose according to the Clebsch-Gordan formula,

$$
\mathscr{V}_{i} \otimes \mathscr{V}_{j} \cong \mathscr{V}_{i+j} \oplus \mathscr{V}_{i+j-2} \oplus \cdots \oplus \mathscr{V}_{|i-j|}
$$

This can be verified using the character formula (2.1).

The purpose of introducing the transvectants here is to have a concrete choice for the isomorphism in Formula (2.7).

Lemma 2.1. The vector space of $\mathbb{C}$-bilinear equivariant maps $\mathscr{V}_{i} \times \mathscr{V}_{j} \rightarrow \mathscr{V}$ has basis $\left\{\langle\cdot, \cdot\rangle_{p}\right\}$, where $0 \leqq p \leqq \min (i, j)$.

Proof. For $p$ in said range, the map $\langle\cdot, \cdot\rangle_{p}: \mathscr{V}_{i} \times \mathscr{V}_{j} \rightarrow \mathscr{V}_{i+j-2 p}$ is non-zero. This can be verified using Formula (2.3), by checking for example that $\left\langle x^{i}, y^{j}\right\rangle_{p} \neq 0$. The statement of the lemma now follows from Schur's lemma and the Clebsch-Gordan formula (2.7). 


\section{ADHM Monads}

We review here briefly the ADHM correspondence between instantons and monads. We refer to the original paper [2] and the books $[5,16]$ for the detailed description of the correspondence.

The first step, as outlined in the introduction, is to translate the problem about instantons on $S^{4}$ to one about holomorphic vector bundles over $\mathbb{C} P^{3}$. This is done using the twistor fibration. To fix our notation we shall quickly describe it now.

Let us denote by $\mathbb{H}$ the quaternionic numbers and consider $\mathbb{H}^{2}$ as a right $\mathbb{H}$ vector space. Restricting scalar multiplication to the complex numbers $\mathbb{C} \subset \mathbb{H}$ makes $\mathbb{H}^{2}$ into a 4-dimensional complex vector space. Mapping a complex line in $\mathbb{H}^{2}$ to the unique $\mathbb{H}$-line containing it defines the Penrose twistor fibration $\pi: \mathbb{C} P^{3} \rightarrow$ $\mathbb{H} P^{1}$, where $\mathbb{H} P^{1}$ is the quaternionic projective line (the space of 1-dimensional quaternionic vector subspaces in $\mathbb{H}^{2}$ ). $\mathbb{H} P^{1}$ can be identified with $S^{4}$ in a way which preserves all relevant structures, see [5], and we shall henceforth assume such an identification $\mathbb{H} P^{1} \cong S^{4}$.

Now, given an hermitian vector bundle $E \rightarrow S^{4}$, the anti-self-duality for the curvature of a connection on $E$ is equivalent to its pull-back on $\mathbb{C} P^{3}$ being of type $(1,1)$, i.e., defining a holomorphic structure on $\pi^{*} E$. Conversely, given a holomorphic vector bundle over $\mathbb{C} P^{3}$ satisfying certain conditions, one can show that it comes from $S^{4}$ in the manner just described.

The next step consists of constructing all holomorphic vector bundles over $\mathbb{C} P^{3}$ that come from instantons on $S^{4}$. They all turn out to arise from a certain monad construction, the $A D H M$ construction, which we now describe.

The monads corresponding to instantons on a rank-2 bundle $E \rightarrow S^{4}$ with second Chern number $c_{2}(E)=k \geqq 0$ are defined by the following data:

(1) A complex vector space $W$ of dimension $k$ equipped with a real structure. This means that an anti-linear map $\sigma$ is defined on $W$ such that $\sigma^{2}=1$.

(2) A complex vector space $V$ of dimension $2 k+2$ equipped with a quaternionichermitian structure. This means that on $V$ are defined an anti-linear map $\sigma$ such that $\sigma^{2}=-1$, and an anti-symmetric $\mathbb{C}$-bilinear form $\langle\cdot, \cdot\rangle$ such that $\left(v, v^{\prime}\right):=\left\langle\sigma v, v^{\prime}\right\rangle$ is a positive-definite hermitian inner product.

Thus we can identify $W$ with $\mathbb{C}^{k}$ endowed with its standard real structure (conjugation), and $V$ with $\mathbb{H}^{k+1} \cong \mathbb{C}^{2 k+2}$ endowed with its standard quaternionic structure (right multiplication by $j$ ) and hermitian inner product $\left(v, v^{\prime}\right)$.

(3) A $\mathbb{C}$-bilinear map

$$
A: W \times \mathbb{C}^{4} \rightarrow V
$$

satisfying the following three "ADHM conditions":

a) The "injectivity condition": $A(w, z)=0$ only if $w=0$ or $z=0$.

b) The "isotropy condition": $\left\langle A(w, z), A\left(w^{\prime}, z\right)\right\rangle=0$ for all $z \in \mathbb{C}^{4}$ and $w, w^{\prime} \in W$.

c) The "reality condition": $\sigma A(w, z)=A(\sigma w, \sigma z)$, where $\mathbb{C}^{4} \cong \mathbb{H}^{2}$ is equipped with its standard quaternionic structure.

An ADHM monad $A: W \times \mathbb{C}^{4} \rightarrow V$ defines a holomorphic vector bundle over $\mathbb{C} P^{3}$ and a corresponding instanton on $S^{4}$ as follows: The injectivity condition means that for every non-zero $z \in \mathbb{C}^{4}$ the image $U_{z}$ of $A(\cdot, z): W \rightarrow V$ is a $k$-dimensional subspace of $V$, and the isotropy condition means that $U_{z}$ is an isotropic subspace 
of $V$ with respect to the symplectic form $\langle\cdot, \cdot\rangle$; thus $U_{z} \subset U_{z}^{\circ}$, where $U_{z}^{\circ}$ is the annihilator of $U_{z}$ with respect to the symplectic form, and $\tilde{E}=U^{\circ} / U$ is the required rank-2 holomorphic vector bundle over $\mathbb{C} P^{3}$. Furthermore, the reality condition ensures that $\tilde{E} \rightarrow \mathbb{C} P^{3}$ descends to a bundle $E \rightarrow S^{4}$ via the twistor fibration, and the hermitian structure $(\cdot, \cdot)$ on $V$ induces an hermitian connection on $E$ which turns out to be anti-self-dual. This is the instanton that corresponds to an ADHM monad.

Two monads $A: W \times \mathbb{C}^{4} \rightarrow V$ and $\tilde{A}: \tilde{W} \times \mathbb{C}^{4} \rightarrow \tilde{V}$ are said to be equivalent if there exist complex-linear isomorphisms $W \rightarrow \tilde{W}$ and $V \rightarrow \tilde{V}$, respecting all structures, and taking $A$ to $\tilde{A}$

The classification theorem of instantons according to ADHM is then:

Theorem 3.1 (ADHM). Every instanton on $S^{4}$ (up to gauge equivalence) is obtained by the ADHM construction from a unique ADHM monad (up to equivalence).

The proof of this theorem relies on an "inverse construction" which associates to each instanton an ADHM monad that gives rise to the instanton. This involves pulling-back the instanton from $S^{4}$ to $\mathbb{C} P^{3}$ (via the twistor fibration) and interpreting the vector spaces $W$ and $V$ and the map $A$ of the required ADHM monad in terms of sheaf cohomology groups associated with the corresponding holomorphic vector bundle on $\mathbb{C} P^{3}$. One can then use twistor methods to interpret part of this data, namely the vector spaces $W$ and $V$, directly in terms of the differential geometry of the instanton on $S^{4}$ :

Theorem 3.2 ([5], chapter 6; [15]). Given an instanton connection on an hermitian vector bundle $E \rightarrow S^{4}$, the vector spaces $W$ and $V$ of the corresponding $A D H M$ monad can be identified with kernels of Dirac operators as follows: Let us fix a spin structure on $S^{4}$ (there is a unique one up to isomorphism) and let $S=S^{+} \oplus S^{-}$ be the corresponding spinor bundle. Then $W$ can be naturally identified with the dual space of E-valued negative harmonic spinors on $S^{4}$,

$$
W^{*} \cong \operatorname{Ker}\left[D_{1}: \Gamma\left(E \otimes S^{-}\right) \rightarrow \Gamma\left(E \otimes S^{+}\right)\right],
$$

where $D_{1}$ is the Dirac operator $\Gamma\left(S^{-}\right) \rightarrow \Gamma\left(S^{+}\right)$coupled to the connection on $E$. Similarly, $V$ can be naturally identified with the space of $E \otimes S^{-}$-valued negative harmonic spinors on $S^{4}$,

$$
V \cong \operatorname{Ker}\left[D_{2}: \Gamma\left(\left(E \otimes S^{-}\right) \otimes S^{-}\right) \rightarrow \Gamma\left(\left(E \otimes S^{-}\right) \otimes S^{+}\right)\right],
$$

where $D_{2}$ is the Dirac operator coupled to the connection on $E \otimes S^{-}$.

For our purposes in this paper we need $G$-equivariant versions of these two theorems. These follow rather easily from the functorial properties of the constructions involved. We now outline the details.

Let us suppose that $E \rightarrow S^{4}$ is a $G$-equivariant hermitian vector bundle, where the group $G$ acts on $E$ by unitary bundle maps covering a conformal orientationpreserving $G$-action on $S^{4}$, so it makes sense to speak about $G$-invariant instantons on $E$. The $G$-action on $S^{4}$ then naturally lifts, via the twistor fibration, to a fiber-preserving action on $\mathbb{C} P^{3}$ (a homomorphism $G \rightarrow P G L(2, \mathbb{H}) \cong S O(4,1)$ ). We further need a lift of the $G$-action to a $\mathbb{H}$-linear action on $\mathbb{H}^{2}$ (a homomorphism $G \rightarrow G L(2, \mathbb{H}))$. 
Remark. If no such lift exists, replacing $G$ by an appropriate double-cover guarantees the existence of a lift. If more than one lift exists, choose arbitrarily. If $G$ is simply-connected, as in our case of $G=S U(2)$, there exists a unique such lift.

Next, a $G$-invariant ADHM monad is defined to be an ADHM monad $A: W \times$ $\mathbb{C}^{4} \rightarrow V$, where $W, V$ and $\mathbb{C}^{4}$ are equipped with a $G$-action respecting all their structures, and where the bilinear map $A$ is $G$-equivariant. Two $G$-invariant ADHM monads are said to be $G$-equivalent if they are equivalent via $G$-equivariant maps. Similarly, $G$-equivalence of $G$-invariant instantons means equivalence by bundle automorphisms commuting with the $G$-action.

Given a $G$-equivariant bundle $E \rightarrow S^{4}$ together with a lift of the $G$-action to $\mathbb{H}^{2}$, the functoriality of the constructions used in proving Theorems 3.1 then yields:

Corollary 3.3. Every G-invariant instanton (up to G-equivalence) is obtained by the ADHM construction from a unique G-invariant monad (up to G-equivalence), where the G-action on $\mathbb{H}^{2}$ is specified by the lift.

We now formulate a $G$-equivariant version of Theorem 3.2. The $G$-action on $\mathbb{H}^{2}$ induces a lift of the $G$-action from $S^{4}$ to the spinor bundles $S^{ \pm}$. If $G$ acts isometrically on $S^{4}$, then the action on $S^{ \pm}$is unitary, and the Dirac operators in Theorem 3.2 are $G$-invariant. (If $G$ acts on $S^{4}$ conformally but not isometrically, which is not our case, then one must include appropriate conformal weights, see [15]). We thus obtain:

Corollary 3.4. The kernels of the Dirac operators in Theorem 3.2 are G-invariant and the identifications in Formulae (3.1) and (3.2) are G-equivariant.

Using this last corollary, together with a $G$-equivariant index theorem and a vanishing theorem for the Dirac operator, one can compute the $G$-representations $W$ and $V$ for the ADHM monad of a $G$-invariant instanton. This will be done in the next section for our case of instantons with quadrupole symmetry.

\section{4. $S U(2)-$ Invariant ADHM Monads}

Following Corollaries 3.3 and 3.4 of the previous section, the classification of instantons with quadrupole symmetry amounts to the classification of $S U(2)$-invariant ADHM monads $A: W \times \mathbb{C}^{4} \rightarrow V$, where the $S U(2)$-action on $\mathbb{C}^{4} \cong \mathbb{H}^{2}$ induces the quadrupole $S U(2)$-action on $S^{4} \cong \mathbb{H} P^{1}$ described in the Introduction. In this section we begin with the determination of the $S U(2)$-action on $\mathbb{C}^{4}, W$ and $V$. Similar computations appear in [7] and in [17].

Recall from the Introduction that rank-2 vector bundles over $S^{4}$ with quadrupole symmetry are classified by a pair $\left(n_{+}, n_{-}\right)$of positive odd integers (see the Appendix for details). Further, recall from the Introduction that a necessary condition for such a bundle to admit a symmetric instanton is $n_{-}=1$.

Proposition 4.1. An instanton with quadrupole symmetry of type $\left(n_{+}, 1\right)$, where $n_{+}=1,3,5 \ldots$, corresponds to an $S U(2)$-invariant $A D H M$ monad $A: W \times \mathbb{C}^{4} \rightarrow V$ 
with the following $S U(2)$-action (denoting $n_{+}$by $n$ for simplicity):

$$
\begin{aligned}
& \mathbb{C}^{4} \cong \mathscr{V}_{3}, \\
& W \cong \mathscr{V}_{n-3} \oplus \mathscr{V}_{n-7} \oplus \mathscr{V}_{n-11} \oplus \ldots, \\
& V \cong \mathscr{V}_{n} \oplus \mathscr{V}_{n-4} \oplus \mathscr{V}_{n-6} \oplus \mathscr{V}_{n-8} \oplus \ldots .
\end{aligned}
$$

Remark. Note that the decomposition of $V$ starts with a "gap" of length 4, and then proceeds in steps of 2 . We have for the first few values of $n_{+}$:

\begin{tabular}{lll}
$n_{+}$ & $W$ & $V$ \\
\hline 1 & $\{0\}$ & $\mathscr{V}_{1}$ \\
3 & $\mathscr{V}_{0}$ & $\mathscr{V}_{3}$ \\
5 & $\mathscr{V}_{2}$ & $\mathscr{V}_{5} \oplus \mathscr{V}_{1}$ \\
7 & $\mathscr{V}_{4} \oplus \mathscr{V}_{0}$ & $\mathscr{V}_{7} \oplus \mathscr{V}_{3} \oplus \mathscr{V}_{1}$ \\
9 & $\mathscr{V}_{6} \oplus \mathscr{V}_{2}$ & $\mathscr{V}_{9} \oplus \mathscr{V}_{5} \oplus \mathscr{V}_{3} \oplus \mathscr{V}_{1}$ \\
11 & $\mathscr{V}_{8} \oplus \mathscr{V}_{4} \oplus \mathscr{V}_{0}$ & $\mathscr{V}_{11} \oplus \mathscr{V}_{7} \oplus \mathscr{V}_{5} \oplus \mathscr{V}_{3} \oplus \mathscr{V}_{1}$.
\end{tabular}

Proof. Let us first prove the isomorphism $\mathbb{C}^{4} \cong \mathscr{V}_{3}$. In light of Corollary 3.3 and the remark preceding it, we need to prove that the $S U(2)$-action on $\mathrm{H}^{2}$ defined by the quaternionic-hermitian representation $\mathscr{V}_{3}$ projects, via the twistor fibration, to the "quadrupole" action on $S^{4}$. Now we know that the quaternionic-hermitian $S U(2)-$ action on $\mathbf{H}^{2}$ projects to an isometric, hence linear, action on $S^{4}$ (in general $S p(2)$ thus projects to $S O(5)$ ), so it is sufficient to show that this action is not reducible (recall that $\mathbb{R}^{5}$ admits a unique, up to equivalence, irreducible $S U(2)$-action, the "quadrupole action"). But any reducible $S U(2)$-representation on $\mathbb{R}^{5}$ must have a trivial summand, so we only need to check that no fixed points occur on $S^{4}$, or in other words, that there are no $S U(2)$-invariant quaternionic lines in $\mathbb{H}^{2}$. This follows from the irreducibility of the representation $\mathscr{r}_{3}$.

For the calculation of the $S U(2)$-action on $W$ and $V$ we need to make an essential use of the following three tools:

1. The interpretation in Theorem 3.2 and Corollary 3.4 of the $S U(2)$-representations $W$ and $V$ in terms of Dirac operators.

2. A fixed-point formula of Atiyah and Bott, applied to the $S U(2)$-equivariant index of these Dirac operators.

3. A vanishing theorem for solutions of Dirac equations which renders the index calculation effective for our purpose.

These matters are explained in detail in well-known references $[5,1,3]$, so here we shall merely quote from these references the relevant facts for the calculation at hand.

Recall from Theorem 3.2 and Corollary 3.4 the interpretation of $W^{*}$ and $V$ as the kernels of the Dirac operators $D_{1}$ and $D_{2}$, respectively. We can thus determine the decomposition of their kernels into irreducible representations by calculating for each of them the corresponding character (trace), via a fixed-point formula of Atiyah and Bott [1], which we now recall briefly.

The set-up relevant for our case is that of an elliptic differential operator $D: \Gamma\left(F^{\prime}\right) \rightarrow \Gamma\left(F^{\prime \prime}\right)$, where $F^{\prime}$ and $F^{\prime \prime}$ are vector bundles over a compact manifold $M$, together with a bundle map $f: F^{\prime} \rightarrow F^{\prime \prime}$ whose induced action on sections 
$\Gamma\left(F^{\prime}\right) \rightarrow \Gamma\left(F^{\prime \prime}\right)$ commutes with $D$ and all of whose fixed points on $M$ are isolated and non-degenerate. It follows that the kernel and cokernel of $D$ (which are necessarily finite-dimensional vector spaces by ellipticity and compactness) are invariant under $f$, and the formula of Atiyah and Bott expresses the difference of the traces of the $f$-action on these spaces (the $f$-index of $D$ ) in terms of the $f$-action at the fixed points:

$$
\begin{gathered}
\operatorname{index}(f, D):=\operatorname{tr}(f, \operatorname{Ker}(D))-\operatorname{tr}(f, \operatorname{coKer}(D))=\sum_{p} v_{p}, \\
v_{p}:=\frac{\operatorname{tr}\left(f, F_{p}^{\prime}\right)-\operatorname{tr}\left(f, F_{p}^{\prime \prime}\right)}{\operatorname{det}\left(1-d_{p} f\right)},
\end{gathered}
$$

where $p$ ranges over the fixed points of $f$ on $M$ and $d_{p} f$ denotes the action of the derivative of $f$ on the tangent space to $M$ at the fixed-point $p$. We note that the particular form of the differential operator $D$ does not enter this remarkable formula, only the details of the action of $f$ at the fixed points are needed.

To apply this fixed-point formula in our case, we restrict the $S U(2)$-action to a circle subgroup, say, the diagonal subgroup of $S U(2)$. It is easy to see then that there are exactly two (antipodal) fixed points in $S^{4} \subset \mathbb{R}^{5}, q_{+}$and $-q_{+}$(see the Appendix). These are necessarily non-degenerate fixed points as the action is by isometries. It follows that the Atiyah-Bott fixed-point formula can be applied to express the difference of the characters of the $S U(2)$-representations $\operatorname{Ker}\left(D_{i}\right)$ and $\operatorname{coKer}\left(D_{i}\right), i=1,2$, in terms of the action of the circle subgroup on the various bundles at the two fixed points.

We now make use of the vanishing of the cokernels of the differential operators $D_{1}$ and $D_{2}$. This follows, via a Weitzenbock-type formula for the Dirac operator, from the positivity of the curvature of $S^{4}$, the self-duality of $S^{4}$ and the self-duality of the connection on $E$ (see, for example, Sect. 6 of [3]). The fixed-point formula (4.1) thus gives the characters of the $S U(2)$-representations $W^{*}$ and $V$. The action on $W^{*}$ is isomorphic to the action on $W$; see the Remark after Formula (2.6).

We examine the details of the circle group action at the fixed points. This is a routine calculation, whose outcome is summarized in the following table of weights:

$$
\begin{array}{lllll} 
& E & S^{-} & S^{+} & T S^{4} \\
\hline q_{+} & \pm n & \pm 3 & \pm 1 & \pm 2, \pm 4 \\
-q_{+} & \pm 1 & \pm 1 & \pm 3 & \pm 2, \pm 4
\end{array}
$$

We next use this information in the Atiyah-Bott fixed-point formula (4.1) for the calculation of the characters of $W$ and $V$, where we use $z$ to parametrize the diagonal matrix with $(z, \bar{z})$ on the diagonal and $|z|=1$ :

$$
\begin{aligned}
\chi_{W} & =\operatorname{tr}(z, W) \\
& =\frac{\left(z^{n}+\bar{z}^{n}\right)\left(z^{3}+\bar{z}^{3}\right)-\left(z^{n}+\bar{z}^{n}\right)(z+\bar{z})+(z+\bar{z})^{2}-(z+\bar{z})\left(z^{3}+\bar{z}^{3}\right)}{(z-\bar{z})^{2}\left(z^{2}-\bar{z}^{2}\right)^{2}} \\
& =\frac{\left(z^{n}+\bar{z}^{n}\right)-(z+\bar{z})}{(z-\bar{z})\left(z^{2}-\bar{z}^{2}\right)}
\end{aligned}
$$


and

$$
\begin{aligned}
\chi_{V} & =\operatorname{tr}(z, V) \\
& =\frac{\left(z^{n}+\bar{z}^{n}\right)\left(z^{3}+\bar{z}^{3}\right)^{2}-\left(z^{n}+\bar{z}^{n}\right)(z+\bar{z})\left(z^{3}+\bar{z}^{3}\right)+(z+\bar{z})^{3}-(z+\bar{z})^{2}\left(z^{3}+\bar{z}^{3}\right)}{(z-\bar{z})^{2}\left(z^{2}-\bar{z}^{2}\right)} \\
& =\frac{\left(z^{n}+\bar{z}^{n}\right)\left(z^{3}+\bar{z}^{3}\right)-(z+\bar{z})^{2}}{(z-\bar{z})\left(z^{2}-\bar{z}^{2}\right)} .
\end{aligned}
$$

Finally, with the Weyl integration formula, we decompose the above expressions for the characters of $V$ and $W$ into sums of characters of irreducible $S U(2)$ representations

$$
\begin{aligned}
& \chi_{W}=\chi_{n-3}+\chi_{n-7}+\ldots, \\
& \chi_{V}=\chi_{n}+\chi_{n-4}+\chi_{n-6}+\ldots,
\end{aligned}
$$

where $\chi_{d}$ is the character of the $(d+1)$-dimensional irreducible $S U(2)$-representation $\mathscr{V}_{d}$, as given in Formula (2.1). We have obtained the decompositions in the statement of the proposition.

\section{The $S U(2)$-Equivariant ADHM Conditions}

The next two sections form the most technical part of the paper and consist of the determination of the $S U(2)$-equivariant $\mathrm{ADHM}$ maps $A: W \times \mathbb{C}^{4} \rightarrow V$, with the $S U(2)$-action on $\mathbb{C}^{4}, W$ and $V$ as given in Proposition 4.1 of the previous section. A fortuitous property of these representations is that they are multiplicity-free, i.e., in their decomposition into irreducible summands each $\mathscr{V}_{d}$ appears at most once.

Let us first establish some notation. For an instanton with quadrupole symmetry of type $\left(n_{+}, 1\right)$, denote the odd integer $n_{+}$by $2 m+1$ and let

$$
A_{l}: \mathscr{V}_{2 l} \times \mathscr{V}_{3} \rightarrow V
$$

$l=m-1, m-3, \ldots$ (ending with $l=0$ or 1 depending on the parity of $m$ ), be the restriction of the bilinear map $A: W \times \mathscr{V}_{3} \rightarrow V$ corresponding to the summand $\mathscr{V}_{2 l} \subset W$ in the decomposition of Proposition 4.1:

$$
W \cong \mathscr{V}_{2(m-1)} \oplus \mathscr{V}_{2(m-3)} \oplus \cdots
$$

The Clebsch-Gordan decompositions

$$
\begin{aligned}
& \mathscr{V}_{2 l} \otimes \mathscr{V}_{3} \cong \mathscr{V}_{2 l+3} \oplus \mathscr{V}_{2 l+1} \oplus \mathscr{V}_{2 l-1} \oplus \mathscr{V}_{2 l-3} \quad \text { for } l \geqq 2, \\
& \mathscr{V}_{2} \otimes \mathscr{V}_{3} \cong \mathscr{V}_{5} \oplus \mathscr{V}_{3} \oplus \mathscr{V}_{1}, \\
& \mathscr{V}_{0} \otimes \mathscr{V}_{3} \cong \mathscr{V}_{3},
\end{aligned}
$$

and Lemma 2.1 allow us to write $A_{l}$ uniquely as a linear combination of transvectants

$$
\begin{aligned}
& A_{l}=a_{l, 0}\langle\cdot, \cdot\rangle_{0}+a_{l, 1}\langle\cdot, \cdot\rangle_{1}+a_{l, 2}\langle\cdot, \cdot\rangle_{2}+a_{l, 3}\langle\cdot, \cdot\rangle_{3} \text { for } l \geqq 2, \\
& A_{1}=a_{1,0}\langle\cdot, \cdot\rangle_{0}+a_{1,1}\langle\cdot, \cdot\rangle_{1}+a_{1,2}\langle\cdot, \cdot\rangle_{2}, \\
& A_{0}=a_{0,0}\langle\cdot, \cdot\rangle_{0} .
\end{aligned}
$$


We call the complex numbers $\left\{a_{l, p}\right\}$ the coefficients of the $S U(2)$-invariant monad. Note, by Proposition 4.1, that $a_{m-1,1}=0$ because of the "gap" $\mathscr{V}_{2 m-1} \not \subset V$, but any other coefficient may be non-zero. It is clear that an $S U(2)$-invariant monad is determined uniquely by its coefficients.

We now formulate the ADHM conditions for an SU(2)-invariant monad in terms of its coefficients.

Theorem 5.1. Let $m$ be a nonnegative integer, and let $n_{+}=2 m+1$. For an SU(2)equivariant bilinear map $A: W \times \mathbb{C}^{4} \rightarrow V$, where the $S U(2)$-action on the vector spaces is as stated in Proposition 4.1, the ADHM conditions of Sect. 3 are equivalent to the following conditions on the coefficients $\left\{a_{l, p}\right\}$ :

a) The $A D H M$ reality condition is equivalent to $a_{l, p} \in \mathbb{R}$ for all $l$ and $p$.

b) The ADHM injectivity condition is equivalent to $a_{l, 0} \neq 0$ for all $l$.

c) The ADHM isotropy condition is equivalent to the following two conditions:

i) The "diagonal isotropy condition":

$$
\begin{aligned}
& a_{l, 2}^{2}=\frac{(2 l+1)}{(2 l-1)^{2}} a_{l, 0}^{2}+\frac{2 l(2 l-3)}{(2 l-1)^{2}} a_{l, 1}^{2} \quad \text { for all } l \geqq 1, \\
& a_{l, 3}^{2}=\frac{(2 l+2)(2 l+5)}{(2 l-1)^{2}} a_{l, 0}^{2}-\frac{9(2 l+1)}{(2 l-1)^{2}} a_{l, 1}^{2} \quad \text { for all } l \geqq 2 ;
\end{aligned}
$$

ii) The "off-diagonal isotropy condition":

$$
a_{l, 0} a_{l+2,2}=a_{l, 1} a_{l+2,3} \quad \text { for all } 1 \leqq l \leqq m-3 .
$$

Proof of 5.1 a) and $b$ ).

a) Follows immediately from the $\mathbb{C}$-antilinearity of $\sigma$ and its compatibility with transvectants (Eq. (2.5)).

b) Suppose the ADHM injectivity condition holds for the $S U(2)$-invariant monad $A: W \times \mathscr{V}_{3} \rightarrow V$. Let $w=x^{2 l} \in \mathscr{V}_{2 l} \subset W$ and let $z=x^{3} \in \mathscr{V}_{3}$. Then $\langle w, z\rangle_{0}=x^{2 l+3} \neq 0$, while $\langle w, z\rangle_{p}=0$ for any $p \geqq 1$. Therefore $A(w, z)=0$ iff $a_{l, 0}=0$, so we have proved that ADHM injectivity implies $a_{l, 0} \neq 0$ for all $l$.

Conversely, suppose that $a_{l, 0} \neq 0$ for all $l$, and that $A(w, z)=0$ for some $w \in W$ and some non-zero $z \in \mathscr{V}_{3}$. We need to prove that $w=0$. Using the direct sum decomposition of $W$ (Eq. (5.1)), write $w=w_{m-1}+w_{m-3}+\cdots$, where $w_{l} \in \mathscr{V}_{2 l} \subset W$. Our assumptions imply that $\left\langle w_{m-1}, z\right\rangle_{0}=0$, but since the $0^{\text {th }}$ transvectant is just multiplication of polynomials, this implies $w_{m-1}=0$. We now proceed by induction. If $l \leqq m-3$ and $w_{l+2}=0$, our assumptions imply that $\left\langle w_{l}, z\right\rangle_{0}=0$, but this again implies $w_{l}=0$. So we have now proved that if $a_{l, 0} \neq 0$ for all $l$, then the ADHM injectivity condition holds.

To prove part c), we first note that the isotropy condition can be reformulated as follows: associate to a monad $A$ the $\mathbb{C}$-quadrilinear form

$$
\begin{aligned}
I_{A}: W \times W \times \mathbb{C}^{4} \times \mathbb{C}^{4} & \rightarrow \mathbb{C}, \\
\left(w, w^{\prime}, z, z^{\prime}\right) & \mapsto\left\langle A(w, z), A\left(w^{\prime}, z^{\prime}\right)\right\rangle .
\end{aligned}
$$

Then the isotropy condition

$$
I_{A}\left(w, w^{\prime}, z, z\right)=0, \quad w, w^{\prime} \in W, z \in \mathbb{C}^{4},
$$


means that for fixed $w, w^{\prime}$ the bilinear form $I_{A}\left(w, w^{\prime}, \cdot, \cdot\right)$ is anti-symmetric, so that its symmetrization in the last two arguments vanishes,

$$
I_{A}^{+}:=I_{A}\left(w, w^{\prime}, z, z^{\prime}\right)+I_{A}\left(w, w^{\prime}, z^{\prime}, z\right)=0 .
$$

Next, we define the $\mathbb{C}$-quadrilinear forms

$$
\begin{aligned}
F_{l, k, p}: \mathscr{V}_{2 l} \times \mathscr{V}_{2 k} \times \mathscr{V}_{3} \times \mathscr{V}_{3} & \rightarrow \mathbb{C}, \\
\left(w, w^{\prime}, z, z^{\prime}\right) & \mapsto\left\langle\langle w, z\rangle_{p+l-\min (l, k)},\left\langle w^{\prime}, z^{\prime}\right\rangle_{p+k-\min (l, k)},\right.
\end{aligned}
$$

and their symmetrization on the last two arguments

$$
F_{l, k, p}^{+}\left(w, w^{\prime}, z, z^{\prime}\right):=F_{l, k, p}\left(w, w^{\prime}, z, z^{\prime}\right)+F_{l, k, p}\left(w, w^{\prime}, z^{\prime}, z\right) .
$$

\section{Lemma 5.2.}

a) The vector space $\mathscr{Y}_{l, k}$ of $S U(2)$-invariant $\mathbb{C}$-quadrilinear forms

$$
\mathscr{V}_{2 l} \times \mathscr{V}_{2 k} \times \mathscr{V}_{3} \times \mathscr{V}_{3} \rightarrow \mathbb{C}
$$

has basis $\left\{F_{l, k, p}\right\}$, where $0 \leqq p \leqq \min (l+k, 3)-|l-k|$.

b) If $l \equiv k(\bmod 2)$, then the subspace $\mathscr{Y}_{l, k}^{+} \subset \mathscr{Y}_{l, k}$ of forms which are symmetric in the last two arguments has dimension:

i) $\min (l, 2)$ if $k=l$,

ii) 1 if $|k-l|=2$ and $\min (k, l) \geqq 1$,

iii) 0 in all other cases.

\section{Proof.}

a) Immediate from the Clebsch-Gordan formula (2.7).

b) $\mathscr{Y}_{l, k}^{+}$is the subspace of invariant vectors in $\left(\mathscr{V}_{2 l} \otimes \mathscr{V}_{2 k}\right) \otimes S^{2}\left(\mathscr{V}_{3}\right)$, where the symmetric square $S^{2}\left(\mathscr{V}_{3}\right) \cong \mathscr{V}_{6} \otimes \mathscr{V}_{2}$. The results now follow from the ClebschGordan formula.

It follows that the set $\left\{F_{l, k, p}^{+}\right\}$spans the vector space $\mathscr{Y}_{l, k}^{+}$, but that this set is not linearly independent in general.

Proposition 5.3. If $l \equiv k(\bmod 2)$, then the following relations constitute a basis for the linear dependence of the set $\left\{F_{l, k, p}^{+}\right\}$in $\mathscr{G}_{l, k}^{+}$:

i) The "diagonal relations", if $k=l$ :

$$
\begin{aligned}
& F_{l, l, 0}^{+}= \begin{cases}-\frac{(2 l+1)}{(2 l-1)^{2}} F_{l, l, 2}^{+}-\frac{(2 l+2)(2 l+5)}{(2 l-1)^{2}} F_{l, l, 3}^{+} & \text {if } l \geqq 2 ; \\
-3 F_{l, 1,2}^{+} & \text {if } l=1 ; \\
0 & \text { if } l=0,\end{cases} \\
& F_{l, l, 1}^{+}= \begin{cases}-\frac{2 l(2 l-3)}{(2 l-1)^{2}} F_{l, l, 2}^{+}+\frac{9(2 l+1)}{(2 l-1)^{2}} F_{l, l, 3}^{+} & \text {if } l \geqq 2 ; \\
2 F_{1,1,2}^{+} & \text {if } l=1 ; \\
0 & \text { if } l=0 .\end{cases}
\end{aligned}
$$


ii) The "off-diagonal relations" if $|k-l|=2$ and $k, l \geqq 1$ :

$$
F_{l, k, 0}^{+}=-F_{l, k, 1}^{+} \text {. }
$$

iii) No non-trivial relations in all other cases.

Proof. The number of linear dependence relations is the difference between the dimension of $\mathscr{Y}_{l, k}$ and the dimension of $\mathscr{Y}_{l, k}^{+}$, both of which are computed in Lemma 5.2. To find a basis, we evaluate $F_{l, k, p}^{+}\left(w, w^{\prime}, z, z^{\prime}\right)$ for appropriate choices of arguments.

i) Substituting $w=x^{a} y^{2 l-a}, z=y^{3}, w^{\prime}=\sigma w$ and $z^{\prime}=\sigma z$ into Formulae (5.4), (5.5) and (2.3), we get

$$
\begin{aligned}
F_{l, l, p}^{+}(w, \sigma w, z, \sigma z) & =\left\langle\langle w, z\rangle_{p},\langle\sigma w, \sigma z\rangle_{p}\right\rangle+\left\langle\langle w, \sigma z\rangle_{p},\langle\sigma w, z\rangle_{p}\right\rangle \\
& =\left\langle\langle w, z\rangle_{p}, \sigma\langle w, z\rangle_{p}\right\rangle-\left\langle\langle w, \sigma z\rangle_{p}, \sigma\langle w, \sigma z\rangle_{p}\right\rangle \\
& =\left\|\langle w, \sigma z\rangle_{p}\right\|^{2}-\left\|\langle w, z\rangle_{p}\right\|^{2}, \\
& =\left\|\frac{1}{p !} x^{a} \frac{\partial^{p} y^{2 l-a}}{\partial y^{p}} \frac{\partial^{p} x^{3}}{\partial x^{p}}\right\|^{2}-\left\|\frac{1}{p !} \frac{\partial^{p} x^{a}}{\partial x^{p}} y^{2 l-a} \frac{\partial^{p} y^{3}}{\partial y^{p}}\right\|^{2} .
\end{aligned}
$$

Evaluating this expression with Formula (2.6) for sufficiently many distinct values of $a$ gives a linear system of equations. The diagonal relations form a basis for the space of solutions.

ii) The proof is similar, but even simpler. Since the dimension of the subspace is at most 1 , it suffices to choose any argument for which $F_{l, l+2,0}^{+}$does not vanish.

iii) $F_{l, k, p}^{+}=0$ in all other cases since $\mathscr{Y}_{l, k}^{+}$is zero-dimensional.

Proof of $5.1 \mathrm{c}$ ). The ADHM isotropy condition $I_{A}^{+}=0$ restricted to $\mathscr{V}_{2 l} \times \mathscr{V}_{2 k} \times$ $\mathscr{V}_{3} \times \mathscr{V}_{3}$ is equivalent to

$$
\sum_{p=0}^{\min (l+k, 3)-|l-k|} a_{l, p+l-\min (l, k)} a_{k, p+k-\min (l, k)} F_{l, k, p}^{+}=0 .
$$

By the dimension count of Lemma $5.2 \mathrm{~b}$ ), there are only two non-trivial cases:

i) When $k=l$, Eq. (5.6) reduces to

$$
\sum_{p=0}^{\min (2 l, 3)} a_{l, p}^{2} F_{l, l, p}^{+}=0
$$

This equation is equivalent, by Proposition $5.3 \mathrm{i}$ ), to the diagonal isotropy condition of Theorem $5.1 \mathrm{c}$ )i).

ii) When $|k-l|=2$ and $\min (k, l) \geqq 1$, then either $k=l+2 \geqq 3$ and Eq. (5.6) reduces to

$$
\sum_{p=0}^{1} a_{l, p} a_{l+2, p+2} F_{l, l+2, p}^{+}=0,
$$

or $l=k+2 \geqq 3$ and Eq. (5.6) reduces to a similar equation. In either case the equation is equivalent, by Proposition 5.3 ii), to the off-diagonal isotropy condition of Theorem $5.1 \mathrm{c}) \mathrm{ii}$ ). 
Corollary 5.4. Suppose the coefficients $\left\{a_{l, p}\right\}$ satisfy the equivariant ADHM conditions. Then

for all $1 \leqq k \leqq m-3$.

$$
\frac{a_{k, 1}}{a_{k, 0}}=\frac{a_{k+2,2}}{a_{k+2,3}} \neq 0
$$

Proof. This is non-trivial only if $m \geqq 4$. All $a_{l, p}$ are real by the ADHM reality condition. By the ADHM injectivity condition $a_{k+2,0} \neq 0$, so $a_{k+2,0}^{2}>0$. Substituting into the ADHM diagonal isotropy condition, we obtain $a_{k+2,2}^{2}>0$, so $a_{k+2,2} \neq 0$. Also $a_{k, 0} \neq 0$ by the injectivity condition, so $a_{k, 0} a_{k+2,2} \neq 0$. From the off-diagonal isotropy condition we conclude that $a_{k, 1} \neq 0$ and $a_{k+2,3} \neq 0$. Dividing the off-diagonal isotropy condition by $a_{k, 0} a_{k+2,3}$ gives the result.

\section{Solutions of the $S U(2)$-Equivariant ADHM Conditions}

The existence statement of Theorem 1.1 is established by the following explicit formula.

Proposition 6.1. For every non-negative integer $m$, the coefficients $\left\{a_{l, p}\right\}$ defined by

$$
a_{l, p}=\sqrt{(2 m+1)^{2}\left((2 l+1) p^{2}-(6 l-1) p+6 l-3\right)^{2}-(2 l-1)^{2}(2 l+3)^{2}} \geqq 0,
$$

satisfy the equivariant ADHM conditions of Theorem 5.1.

Proof. The proof consists of merely substituting the coefficients $a_{l, p}$ into the equivariant ADHM conditions of Theorem 5.1. Clearly $a_{m-1,1}=0$. The inequality $l \leqq m-1$ is equivalent to $(2 l+3)^{2} \leqq(2 m+1)^{2}$, and the reality and nonnegativity of the $a_{l, p}$ are immediate from:

$$
\begin{aligned}
& a_{l, 0}^{2}=(2 l-1)^{2}\left(9(2 m+1)^{2}-(2 l+3)^{2}\right)>0, \\
& a_{l, 1}^{2}=(2 l-1)^{2}\left((2 m+1)^{2}-(2 l+3)^{2}\right) \geqq 0, \\
& a_{l, 2}^{2}=(2 l+3)^{2}\left((2 m+1)^{2}-(2 l-1)^{2}\right)>0, \\
& a_{l, 3}^{2}=(2 l+3)^{2}\left(9(2 m+1)^{2}-(2 l-1)^{2}\right)>0 .
\end{aligned}
$$

The first inequality implies the injectivity condition $a_{l, 0} \neq 0$. Verification of the diagonal and off-diagonal isotropy conditions requires a simple calculation.

To prove uniqueness, we need to study the equivalence of $S U(2)$-invariant monads. If $A: W \times \mathbb{C}^{4} \rightarrow V$ is an $S U(2)$-invariant monad then, by definition, any real $S U(2)$-equivariant automorphism $W \rightarrow W$ and unitary quaternionic $S U(2)$ equivariant automorphism $V \rightarrow V$ take $A$ into an $S U(2)$-equivalent $S U(2)$-invariant monad $\tilde{A}$. By Schur's lemma, the restriction of an equivariant real invertible map $W \rightarrow W$ to $\mathscr{V}_{2 l} \subset W$ is the identity map multiplied by a non-zero real number $\gamma_{l}$. Similarly, the restriction of an equivariant unitary quaternionic map $\mathscr{V} \rightarrow \underset{\tilde{A}}{V}$ to $\mathscr{V}_{j} \subset V$ is the identity map multiplied by $\kappa_{j}= \pm 1$. The coefficents $\left\{\tilde{a}_{l, p}\right\}$ of $\tilde{A}$ are then related to the coefficients $\left\{a_{l, p}\right\}$ of $A$ by

$$
\tilde{a}_{l, p}=\kappa_{2 l+3-2 p} \gamma_{l} a_{l, p} \text {. }
$$


Definition 6.2. Let $k$ be an integer. An $S U(2)$-invariant monad $A$ is called $k$ canonical if its coefficients $\left\{a_{l, p}\right\}$ satisfy the following conditions for all $l \geqq k$ :

i) $a_{l, p} \geqq 0$ for all $p$.

ii) $a_{l, 0}=1$.

The uniqueness proof for Theorem (1.1) proceeds by bringing $S U(2)$-invariant ADHM monads into 0-canonical form.

Lemma 6.3. Every $S U(2)$-invariant $A D H M$ monad is $S U(2)$-equivalent to a 0 canonical monad.

Proof. Let $\left\{a_{l, p}\right\}$ be the coefficients of an $S U(2)$-invariant ADHM monad $A$. We shall first construct an $S U(2)$-equivalent $(m-1)$-canonical monad with coefficients $\left\{\tilde{a}_{l, p}\right\}$. If $m=0$, the set of coefficients is empty, so $A$ is trivially 0 -canonical. Suppose $m \geqq 1$. Let $\kappa_{2 m+1}=1$ and $\gamma_{m-1}=1 / a_{m-1,0}$, so $\tilde{a}_{m-1,0}=1$. If $m=1$, we are done. Suppose $m \geqq 2$. As always, we must have $\tilde{a}_{m-1,1}=0$. Choose $\kappa_{2 m-3}$ so that $\tilde{a}_{m-1,2}=\kappa_{2 m-3} \gamma_{m-1} a_{m-1,2} \geqq 0$. If $m=2$, we are done. Suppose that $m \geqq 3$. Choose $\kappa_{2 m-5}$ so that $\tilde{a}_{m-1,3}=\kappa_{2 m-5} \gamma_{m-1} a_{m-1,3} \geqq 0$. Letting $\gamma_{m-3}=\kappa_{2 m-3} / a_{m-3,0}$, we obtain $\tilde{a}_{m-3,0}=1$. If $m=3$, we are done. Suppose $m \geqq 4$. Letting any remaining $\kappa_{j}=$ 1 and $\gamma_{l}=1$, we have constructed an $(m-1)$-canonical $S U(2)$-equivalent monad $\tilde{A}$.

We now use induction on $k$. Given a $(k+2)$-canonical $S U(2)$-invariant ADHM monad $B$ with coefficients $\left\{b_{l, p}\right\}$, we shall construct an $S U(2)$-equivalent $k$ canonical monad $\tilde{B}$ with coefficients $\left\{\tilde{b}_{l, p}\right\}$. Suppose $m \geqq 4$. Let $0 \leqq k \leqq m-3$ with $k \equiv m-1(\bmod 2)$. Let $\kappa_{j}=1$ for $j \geqq 2 k+1$. Let $\gamma_{k}=1 / b_{k, 0}$, and $\gamma_{l}=1$ for $l>k$. Then $\tilde{b}_{k, 0}=1$. If $k=0$, we are done. Assume that $k \geqq 1$. Since $B$ is $(k+2)$ canonical $b_{k+2,2}$ and $b_{k+2,3}$ are positive, and Corollary 5.4 implies $b_{k, 1} / b_{k, 0}>0$, proving the positivity of $\tilde{b}_{k, 1}=\gamma_{k} b_{k, 1}=b_{k, 1} / b_{k, 0}$. Choose $\kappa_{2 k-1}$ so that $\tilde{b}_{k, 2} \geqq 0$. If $k=1$, we are done. Assume $k \geqq 2$. Choose $\kappa_{2 k-3}$ so that $\tilde{b}_{k, 3} \geqq 0$. Letting any remaining $\kappa_{j}=1$ and $\gamma_{l}=1$, we have constructed a $k$-canonical $S U(2)$-equivalent $\operatorname{monad} \tilde{B}$.

Lemma 6.4. For any non-negative integer $m$, there is at most one 0-canonical invariant $A D H M$ monad.

Proof. For a given $m$, suppose that $\left\{a_{l, p}\right\}$ and $\left\{\tilde{a}_{l, p}\right\}$ are both coefficients of invariant 0 -canonical $\mathrm{ADHM}$ monads. We shall show that $\tilde{a}_{l, p}=a_{l, p}$ for all $l$ and $p$. If $m=0$, the statement is trivial. Suppose $m \geqq 1$. Because both are 0 canonical, $\tilde{a}_{m-1,0}=a_{m-1,0}=1$. If $m=1$, we are done. Suppose $m \geqq 2$. We always have $\tilde{a}_{m-1,1}=a_{m-1,1}=0$. By the diagonal isotropy condition of Theorem 5.1 c)i), $\tilde{a}_{m-1,2}^{2}=a_{m-1,2}^{2}$, so $\tilde{a}_{m-1,2}=a_{m-1,2}$ by non-negativity. If $m=2$, we are done. Suppose $m \geqq 3$. Again by the diagonal isotropy condition and non-negativity, $\tilde{a}_{m-1,3}=a_{m-1,3}$. If $m=3, \tilde{a}_{0,0}=a_{0,0}=1$ and we are done.

Suppose $m \geqq 4$. Let $0 \leqq k \leqq m-3$ with $k=m-1(\bmod 2)$. We shall show that if $\tilde{a}_{k+2, p}=a_{k+2, p}$ for all $p$, then $\tilde{a}_{k, p}=a_{k, p}$ for all $p$. Induction on $k$ then completes the proof of the lemma. If $k=0$, only $p=0$ occurs, and the statement is trivial because $\tilde{a}_{0,0}=a_{0,0}=1$. Assume $k \geqq 1$. Since both monads are 0 -canonical, Corollary 5.4 implies $a_{k, 1}=a_{k+2,2} / a_{k+2,3}$ and $\tilde{a}_{k, 1}=\tilde{a}_{k+2,2} / \tilde{a}_{k+2,3}$. By assumption $\tilde{a}_{k+2, p}=a_{k+2, p}$ for all $p$, so $\tilde{a}_{k, 1}=a_{k, 1}$. Using this and $\tilde{a}_{k, 0}=a_{k, 0}=1$ in the diagonal isotropy condition gives $\tilde{a}_{k, 2}^{2}=a_{k, 2}^{2}$, and also $\tilde{a}_{k, 3}^{2}=a_{k, 3}^{2}$ if $k \geqq 2$. From non-negativity we conclude that $\tilde{a}_{k, p}=a_{k, p}$ for all $p$. 
The uniqueness statement of Theorem 1.1 follows:

Corollary 6.5. For any non-negative integer $m$, there is at most one $S U(2)-$ equivalence class of SU(2)-invariant ADHM monads, hence at most one gauge equivalence class of SU(2)-invariant instantons on the bundle of symmetry type $\left(n_{+}, n_{-}\right)=(2 m+1,1)$.

Proof. For a given $m$, suppose $A$ and $B$ are $S U(2)$-invariant ADHM monads. By Lemma 6.3, there exist 0-canonical $S U(2)$-invariant ADHM monad $\tilde{A}$ and $\tilde{B}$ that are $S U(2)$-equivalent to $A$ and $B$, respectively. By Lemma 6.4, $\tilde{A}=\tilde{B}$. Therefore $A$ and $B$ are $S U(2)$-equivalent, and correspond, by Corollary 3.3, to instantons which are $S U(2)$-equivalent - hence gauge equivalent.

Note that the $S U(2)$-invariant ADHM monads of Proposition 6.1 are not 0-canonical.

\section{Examples}

We exhibit the first few examples, using Lemma 6.3 to bring the ADHM monads of Proposition 6.1 into 0-canonical form.

The invariant monad with $m=0$, and $\left(n_{+}, n_{-}\right)=(1,1)$, is trivial, corresponding to the flat connection on the trivial bundle $c_{2}=0 . W=\{0\}$, so $A$ is the zero map, and the set of coefficients is empty.

The invariant monad with $m=1$, and $\left(n_{+}, n_{-}\right)=(3,1)$, correpsonds to the standard ("SO(5)-invariant") instanton on the bundle with $c_{2}=1$. The monad is described by the single coefficient $a_{0,0}=1$. Neither the diagonal nor the off-diagonal isotropy condition enters.

The invariant monad with $m=2$, and $\left(n_{+}, n_{-}\right)=(5,1)$, has $c_{2}=3$, and has coefficients:

$$
a_{1,0}=1, \quad a_{1,1}=0, \quad a_{1,2}=\sqrt{3} .
$$

The diagonal isotropy condition enters, but the off-diagonal does not.

The invariant monad with $m=3$, and $\left(n_{+}, n_{-}\right)=(7,1)$, has $c_{2}=6$, and has coefficients:

$$
\begin{gathered}
a_{2,0}=1, \quad a_{2,1}=0, \quad a_{2,2}=\sqrt{5 / 9}, \quad a_{2,3}=\sqrt{6}, \\
a_{0,0}=1 .
\end{gathered}
$$

Both the diagonal and off-diagonal isotropy conditions enter in this, and all following monads.

The invariant monad with $m=4$, and $\left(n_{+}, n_{-}\right)=(9,1)$ has $c_{2}=10$, and has coefficients:

$$
\begin{gathered}
a_{3,0}=1, \quad a_{3,1}=0, \quad a_{3,2}=\sqrt{7 / 25}, \quad a_{3,3}=\sqrt{88 / 25}, \\
a_{1,0}=1, \quad a_{1,1}=\sqrt{7 / 88}, \quad a_{1,2}=\sqrt{125 / 44} .
\end{gathered}
$$

The invariant monad with $m=5$, and $\left(n_{+}, n_{-}\right)=(11,1)$, has $c_{2}=15$, and has coefficients:

$$
\begin{gathered}
a_{4,0}=1, \quad a_{4,1}=0, \quad a_{4,2}=\sqrt{9 / 49}, \quad a_{4,3}=\sqrt{130 / 49}, \\
a_{2,0}=1, \quad a_{2,1}=\sqrt{9 / 130}, \quad \begin{array}{c}
a_{2,2}=\sqrt{343 / 585}, \quad a_{2,3}=\sqrt{147 / 26}, \\
a_{0,0}=1 .
\end{array}
\end{gathered}
$$




\section{Appendix}

The purpose of this appendix is to present the classification of rank-2 hermitian vector bundles over $S^{4}$ with quadrupole symmetry, in terms of a pair of positive odd integers $\left(n_{+}, n_{-}\right)$, as mentioned in the Introduction. The reference here is Sect. 2 of [10].

The idea is a variant of the usual "clutching" construction for vector bundles on a sphere. We recall that this construction is based on viewing the sphere as a union of two hemispheres (disks), intersecting along an equator (a sphere of one dimension lower); since each hemisphere is homotopically trivial (contractible) any bundle over it is trivial, and so any bundle over the sphere can be obtained by the "gluing" of trivial bundles over the hemispheres via a map from the equator to the structure group (the unitary group for hermitian bundles).

In our case of bundles with "quadrupole symmetry," i.e. $S U(2)$-equivariant hermitian vector bundles over $S^{4}$ with the $S U(2)$-action on $S^{4} \subset \mathbb{R}^{5}$ given by the 5-dimensional irreducible representation of $S U(2)$, we have a similar construction. For this we need to examine first the orbit structure of the $S U(2)$-action on $S^{4}$. One then checks that (1) a generic orbit is 3-dimensional; (2) there are two singular orbits; and (3) $S^{4}$ is the union of two tubular neighborhoods (equivariant disk bundles), one of each of the two singular orbits, intersecting along a generic orbit.

In general, for a smooth action of a compact group, a tubular-neighborhood of a given orbit can be "radially" contracted, equivariantly, to the orbit. It follows that an equivariant bundle over such a neighborhood is the pull-back, under the contraction, of an equivariant bundle over the orbit, i.e. a homogeneous bundle, and thus given as the bundle associated to a representation of the stabilizer group of a point on the orbit. The "gluing" of such bundles over distinct tubular neighborhoods is done via an equivariant isomorphism between the restrictions of the bundles to the intersection of the tubular neighborhoods.

To describe the situation precisely in our case of "quadrupole symmetry" we need to fix first some notation. It is convenient to identify our symmetry group $S U(2)$ with $S p(1)$, the group of unit quaternions. This can be done by, say, considering the quaternions $\mathbb{H}$ as a right $\mathbb{C}$ vector space with a $\mathbb{C}$-basis $\{1, j\}$, and let $\mathrm{Sp}(1)$ act by left multiplication. Next, we take as a model for the 5-dimensional irreducible representation of $\mathrm{Sp}(1)$ the space of traceless real quadratic forms $q$ on the Lie algebra of $\mathrm{Sp}(1)$ (imaginary quaternions), with the $\mathrm{Sp}(1)$-action induced by the adjoint action on the Lie algebra, and norm given by $\|q\|^{2}=\operatorname{tr}\left(q^{2}\right)$. The $\mathrm{Sp}(1)$-action preserves this norm, so we obtain an $\mathrm{Sp}(1)$ action on the unit sphere $S^{4}$. More explicitly, if we fix a basis for the Lie algebra of $\operatorname{Sp}(1)$, say $\{i, j, k\}$, with the dual basis $\{\alpha, \beta, \gamma\}$, then the coefficients of a traceless quadratic form $q$ constitute a $3 \times 3$ traceless symmetric matrix, so that the $\operatorname{Sp}(1)$-action factors through the usual conjugation action of $S O(3)$ on the space of symmetric matrices.

The orbits of the $\mathrm{Sp}(1)$-action are parametrized by the set of 3 eigenvalues of a form. Generically, the 3 eigenvalues are distinct, so the two singular orbits consist exactly of those forms with a degenerate eigenvalue, with the sign of the degenerate eigenvalue distinguishing between the two singular orbits.

Now let us fix two points on $S^{4}$, one on each of the two singular orbits,

$$
q_{+}:=\frac{1}{\sqrt{6}}\left(\alpha^{2}+\beta^{2}-2 \gamma^{2}\right), \quad q_{-}:=\frac{1}{\sqrt{6}}\left(2 \alpha^{2}-\beta^{2}-\gamma^{2}\right),
$$


and denote the corresponding stabilizer groups by $H_{+}$and $H_{-}$, respectively;

$$
H_{+}=\left\{e^{k \theta}\right\} \cup\left\{i e^{k \theta}\right\}, \quad H_{-}=\left\{e^{i \theta}\right\} \cup\left\{k e^{i \theta}\right\}, \quad 0 \leqq \theta<2 \pi .
$$

Then picking a point $q_{0}$ in the intersection of the normal disks at $q_{ \pm}$,

$$
q_{0}=\frac{1}{\sqrt{2}}\left(\alpha^{2}-\gamma^{2}\right)
$$

the stabilizer group at $q_{0}$ is

$$
\Gamma:=H_{+} \cap H_{-}=\{ \pm 1, \pm i, \pm j, \pm k\} .
$$

With this notation understood, the clutching construction for bundles with quadrupole symmetry can now be stated:

Proposition 7.1. There is a bijective correspondence between SU(2)-equivalence classes of SU(2)-equivariant hermitian vector bundles over $S^{4}$ and equivalence classes of triples $\left(\rho_{+}, \rho_{-}, \phi\right)$, where

(1) $\rho_{ \pm}$are linear unitary representations of $H_{ \pm}$on hermitian vector spaces $V_{ \pm}$, respectively;

(2) $\phi$ is a $\Gamma$-equivariant unitary isomorphism $V_{+} \rightarrow V_{-}$; and

(3) two triples $\left(\rho_{+}, \rho_{-}, \phi\right)$ and $\left(\tilde{\rho}_{+}, \tilde{\rho}_{-}, \tilde{\phi}\right)$ are equivalent if there exist $H_{ \pm^{-}}$ equivariant unitary isomorphisms $V_{ \pm} \rightarrow \tilde{V}_{ \pm}$, taking $\phi$ to $\tilde{\phi}$.

The next step then is to study the representations of the stabilizers $H_{ \pm}$and their restriction to $\Gamma$. The calculation is based on the technique of induced representations which we now review briefly.

Let $K$ be a compact Lie group with a subgroup $K^{\prime}$ of finite index. There are two additive maps relating the complex representation rings $R K$ and $R K^{\prime}$,

$$
i^{*}: R K \rightarrow R K^{\prime}
$$

and

$$
i_{*}: R K^{\prime} \rightarrow R K .
$$

The first is restriction $\left.V \mapsto V\right|_{K^{\prime}}$ and the second induction $W \mapsto \operatorname{Maps}_{K^{\prime}}(K, W)=$ $\Gamma\left(K \times_{K^{\prime}} W\right)$. These are related by Frobenius reciprocity

$$
\left(i^{*} V, W\right)=\left(V, i_{*} W\right),
$$

for any $V \in R K$ and $W \in R K^{\prime}$, where $(\cdot, \cdot)$ denotes the standard pairing generated by declaring for two irreducible representations $A$ and $B$ (of the same group) that $(A, B)=1$ if $A$ and $B$ are isomorphic, else $(A, B)=0$. Reciprocity implies that all representations of $K$ can be obtained by induction from $K^{\prime}$ : taking an irreducible $V \in R K$,

$$
\left(i_{*} i^{*} V, V\right)=\left(i^{*} V, i^{*} V\right)>0,
$$

so $V$ can be obtained as one of the irreducible components of the induced representation $i_{*} i^{*} V$.

Now back to the representations of $H_{ \pm}$, beginning with $H_{-}$. We find its representations by inducing from its index-2 subgroup $H_{-}^{\prime}:=\left\{e^{i \theta} \mid 0 \leqq \theta<2 \pi\right\}$. Let $\lambda \in R H_{-}^{\prime}$ denote the basic 1-dimensional representation, then $R H_{-}^{\prime}=\mathbb{Z}\left[\lambda, \lambda^{-1}\right]$. The following is obtained by a calculation using Frobenius reciprocity. 
Proposition 7.2. For all $n, m \in \mathbb{Z}$,

1) $i^{*} i_{*} \lambda^{n} \cong \lambda^{n}+\lambda^{-n}$.

2) $i_{*} \lambda^{n}$ is irreducible if $n \neq 0$.

3) $i_{*} 1=1+\sigma$, where $\sigma$ is the "sign representation" ( 1 on $H_{-}^{\prime}$ and -1 on the other component).

4) $i_{*} \lambda^{n} \cong i_{*} \lambda^{m}$ iff $|n|=|m|$.

Corollary 7.3. A complete list, without repetitions, of irreducible representations of $H_{-}$is given by

$$
1, \sigma, i_{*} \lambda^{n}, \quad n=1,2,3, \ldots .
$$

For $H_{+}$we have the same result with the obvious notational reinterpretation.

Next, we restrict the $H_{ \pm}$representations to the subgroup $\Gamma$. Using a character table for $\Gamma$ we find that the group $\Gamma$ has 5 irreducible representations: $1, \sigma_{1}, \sigma_{2}, \sigma_{3}$, and $\mathbb{C}^{2}$. The first 4 are 1-dimensional: 1 is the trivial, $\sigma_{1}$ is 1 on $\{ \pm 1, \pm i\}$ and -1 on the rest, $\sigma_{2}$ and $\sigma_{3}$ similarly with $j$ and $k$ instead of $i$, respectively. $\mathbb{C}^{2}$ is the restriction to $\Gamma$ of the standard 2-dimensional Sp(1)-representation (denoted by $\mathscr{V}_{1}$ in Sect. 2). A simple calculation then yields:

Proposition 7.4. Upon restriction from $H_{-}$to $\Gamma, \sigma$ restricts to $\sigma_{1}$ and $i_{*} \lambda^{n}$ restricts to

$$
\begin{aligned}
& \mathbb{C}^{2} \text { if } n \text { is odd, } \\
& 1+\sigma_{1} \text { if } n \equiv 0(\bmod 4), \\
& \sigma_{2}+\sigma_{3} \text { if } n \equiv 2(\bmod 4) .
\end{aligned}
$$

The corresponding result for $H_{+}$is

Proposition 7.5. Upon restriction from $H_{+}$to $\Gamma, \sigma$ restricts to $\sigma_{3}$ and $i_{*} \lambda^{n}$ restricts to

$$
\begin{aligned}
& \mathbb{C}^{2} \text { if } n \text { is odd, } \\
& 1+\sigma_{3} \text { if } n \equiv 0(\bmod 4) \\
& \sigma_{1}+\sigma_{2} \text { if } n \equiv 2(\bmod 4)
\end{aligned}
$$

Comparing the last two propositions with Proposition 7.1 we obtain:

Corollary 7.6. A complete list, without repetitions, of equivalence classes of nontrivial triples $\left(\rho_{+}, \rho_{-}, \phi\right)$, with $\operatorname{dim}\left(V_{ \pm}\right)=2$, and thus, according to Proposition 7.1, of non-trivial rank-2 vector bundles over $S^{4}$ with quadrupole symmetry, is given by

$$
\left(i_{*} \lambda^{n+}, i_{*} \lambda^{n-}, 1\right), \quad n_{ \pm}=1,3,5 \ldots
$$

Finally, a word about Chern numbers and our orientation convention. It is rather straightforward to derive a formula that expresses the second Chern number of our bundles with quadrupole symmetry in terms of the integer invariants $n_{+}$and $n_{-}$of the last corollary. This can be done either by an explicit integration of a curvature expression using an invariant connection (as in $[20,4]$ ), or by a localization principle for equivariant cohomology classes (as in [10]). Either way, to arrive at Formula (1.1) in the Introduction for the second Chern number $c_{2}$ one needs to integrate the second Chern class of the bundle over $S^{4}$, so an orientation choice for $S^{4}$ affects the sign of $c_{2}$. We choose the orientation for $S^{4}$ that fixes the sign of $c_{2}$ to be as given in Formula (1.1). 


\section{References}

1. Atiyah, M.F., Bott, R.: A Lefschetz fixed-point formula for elliptic complexes I. Ann. Math. 86, 374-407 (1967)

2. Atiyah, M.F., Drinfeld, V.G., Hitchin, N.J., Manin, Y.I.: Construction of Instantons. Phys. Lett. 65A, 185 (1978)

3. Atiyah, M.F., Hitchin, N.J., Singer, I.M.: Self-Duality in Four Dimensional Riemannian Geometry. Proc. R. Soc. Lond. A 362, 425 (1978)

4. Avron, J.E., Sadun, L., Segert, J., Simon, B.: Chern Numbers, Quaternions, and Berry's Phases in Fermi System. Commun. Math. Phys. 124, 595 (1989)

5. Atiyah, M.F.: Geometry of Yang-Mills Fields. Lezioni Fermiane, Accademia Nazionale dei Lincei and Scuola Normale Superiore, Pisa, 1979

6. Atiyah, M.F.: Magnetic monopoles in hyperbolic space. Vector Bundles on Algebraic Varieties, Proc. of Bombay Colloquium 1984

7. Austin, D.M.: $S O(3)$-Instantons on $L(p, q) \times R$. J. Diff. Geom. 32, 383 (1990)

8. Braam, P.J., Austin, D.M.: Boundary values of hyperbolic monopoles. Nonlinearity 3, 809823 (1990)

9. Bourguignon, J.P., Lawson, J.B.: Stability and isolation phenomena for Yang-Mills equations. Commun. Math. Phys. 79, 189 (1982)

10. Bor, G.: Yang-Mills fields which are not self-dual. Commun. Math. Phys. 145, 393-410 (1992)

11. Bryant, R.L.: Two exotic holonomies in dimensions four, path geometries, and twistor theory. Proc. of Symposia in Pure Mathematics, Vol. 51, 1991, pp. 33-88

12. Furuta, M.: $Z_{a}$-invariant $S U(2)$ instantons over the Four Sphere. In: Geometry of lowdimensional manifolds, 1 (Durham, 1989), London Math. Soc. Lecture Note Ser., 150, Cambridge: Cambridge Univ. Press, 1990, pp. 161-174

13. Furuta, M., Hashimoto, Y.: Invariant instantons on $S^{4}$. J. Fac. Sci. Univ. Tokyo Sect. IA Math. 37, 585-600 (1990)

14. Hitchin, N.J.: Twistor spaces, einstein metrics, and isomonodromic deformations. J. Diff. Geom. 42, 30-112 (1995)

15. Hitchin, N.J.: Linear field equations on self-dual spaces. Proc. R. Soc. Lond. A 370, 173-191 (1980)

16. Manin, Yu.I.: Gauge Field Theory and Complex Geometry. Berlin: Springer-Verlag, 1988

17. Parker, T.H.: A Morse Theory for Equivariant Yang-Mills. Duke Math. J. 66, 337-356 (1992)

18. Sadun, L.: A symmetric family of Yang-Mills field. Commun. Math. Phys. 163, 257-291 (1994)

19. Segert, J.: An equivariant Atiyah-Ward correspondence. In preparation

20. Sadun, L., Segert, J.: Non-self-dual Yang-Mills connections with quadrupole symmetry. Commun. Math. Phys. 145, 363-391 (1992)

21. Sadun, L., Segert, J.: Stationary Points of the Yang-Mills Action. Comm. Pure and Appl. Math. 45, 641-684 (1992)

22. Sibner, L.M., Sibner, R.J., Uhlenbeck, K.: Solutions to Yang-Mills Equations which are not Self-Dual. Proc. Natl. Acad. Sci. USA 86, 8610 (1989)

23. Taubes, C.H.: On the Equivalence of the First and Second order Equations for Gauge Theories. Commun. Math. Phys. 75, 207-227 (1980)

24. Ward, R.S.: On self-dual gauge fields. Phys. Lett. 61A, 81-82 (1977) 
